\title{
93. Rearrangement of Integrated HBV DNA in Descendants of Transgenic Mice
}

\author{
By Okio Hino,*' Kimie Nomura, ${ }^{*}$ Keiko Ohtake,*) \\ Tomoyuki Kitagawa,*) Haruo Sugano,*) Shiari KimuRa,**) \\ Minesuke YoKoYama,**) and Motoya KATSUKI**),***) \\ (Communicated by Toshio Kurokawa, M. J. A., Nov. 12, 1986)
}

Persistent hepatitis B virus (HBV) infection is epidemiologically closely associated with the development of human hepatocellular carcinoma (HCC).1) Furthermore, it has been shown that HBV DNA is integrated at a very high frequency in the host DNA of HCCs developing in carrier patients positive for hepatitis B surface antigen $(\mathrm{HBsAg}) .2-4)$ The question of whether the integration of viral DNA itself acts as complete carcinogenic factor, independent of any indirect co-carcinogenic action associated with virus infection, however, remains unclear.") For example, HBV, so far, has not been shown to contain a typical viral oncogene. Furthermore a promoter insertion mechanism does not appear to be operative, since a common integration site in host chromosomal DNA has not been identified. ${ }^{6)}$ Although the possibility of an X-gene product acting in "trans" on a different chromosome has been suggested, in analogy with leukemogenesis by Human T-cell Lymphotrophic Virus Type I (HTLV-I), 7) supportive evidence is lacking. On the other hand, chromosomal translocation and/or deletion has been shown to frequently occur in association with viral integration, ${ }^{8)-10)}$ and it is well-known that agents which cause chromosomal aberrations are associated with increased risks of cancer.11.1.2) The ability of $\mathrm{HBV}$ to generate chromosomal aberrations may therefore be involved in a direct causal mechanism for HCC generation. In order to develop a laboratory system to scrutinize this possibility, we have constructed transgenic mice with HBV DNA and commenced investigation of integration associated rearrangement.

Materials and methods. HBV DNA having an inverted repeat sequence was obtained from a HCC. ${ }^{10)}$ This viral sequence can form a stem and loop structure through junction of the $\mathrm{X}, \mathrm{S}$ and $\mathrm{C}$ gene regions with the pre $\mathrm{C}$ region including DR1 (Fig. 1). This configuration was used for 4 reasons; first, previous studies demonstrated that when simple linear HBV DNA was microinjected into mouse eggs, no rearrangement was observed (personal communication; Dr. F. V. Chisari, Research Institute of Scripps Clinic, California, Dr. C. Pourcel, Pasteur Institute, France, Dr. R. Burk, Albert Einstein College of Medicine, New York), second, integrated HBV DNAs often have inverted repeat structures, ${ }^{10), 13), 14)}$ third, the DR1 region, which is thought to be the recombination site between viral and host $\mathrm{DNA}^{15)}$ and also the origin of replication, ${ }^{16)}$ is present in the loop

*) Cancer Institute, Department of Pathology, 1-37-1 Kami-ikebukuro, Toshimaku, Tokyo 170 .

**) Central Institute for Experimental Animals, 1430 Nogawa, Miyamae-ku, Kawasaki, Kanagawa 213.

***) Tokai University School of Medicine, Department of Cell Biology and Institute of Medical Sciences, Boseidai, Isehara, Kanagawa 259-11. 

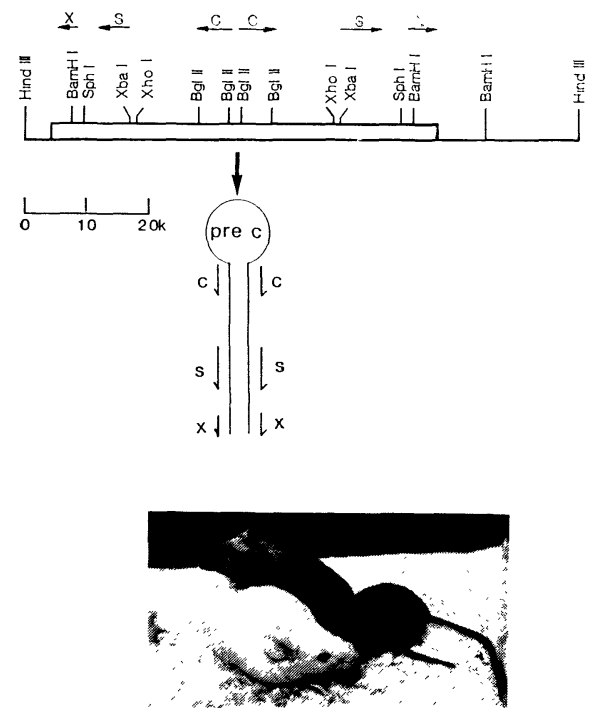

Fig. 2 (above). Transgenic mice ( $\mathrm{C} 3 \mathrm{H}$, agouti) progeny from a pseudopregnant mother $(\mathrm{MCH}$ (ICR) ; albino).

Fig. 3. (right). Southern blot analysis of placenta DNAs from original transgenic mice [(H2-1p( $\hat{o}), \mathrm{H} 2-2 \mathrm{p}(\hat{\delta})$ and $\mathrm{H} 2-5 \mathrm{~m}(q)]$. BamHI : BamHI digested. Hind III: Hind III digested. Positions of molecular weight markers of known length are shown on the right.
Fig. 1 (left). Restriction endonuclease mapping and genetic organization of HBV DNA used for microinjection (BamHI $5.4 \mathrm{~kb}$ fragment). C: HBcAg gene. $\mathrm{S}: \mathrm{HBsAg}$ gene. $\mathrm{X}: \mathrm{X}$ gene. $\mathrm{k}$ : Kilobase.

structure, fourth, in herpesvirus DNA, a relationship between transforming activity and stem and loop structure of the virus has been suggested.17)

The BamHI fragment of this integrated HBV DNA was subcloned as a $5.4 \mathrm{~kb}$ BamHI fragment in the BamHI site of pBR327 vector (designated pBC45.4B). (The pBR327 plasmid was kindly supplied by Dr. J. Fujisawa, Cancer Institute, Dep. of Viral Oncology.) The plasmid pBC4-5.4B was digested with BamHI and the plasmid vector removed by low melting agarose gel electrophoresis. The BamHI fragment without plasmid DNA was purified by phenol and ethanol precipitation, adjusted to $2 \mu \mathrm{g} / \mathrm{ml}$ and then a few hundred molecules of the linearized DNA were microinjected into male pronuclei of 55 fertilized one cell eggs of the $\mathrm{C} 3 \mathrm{H} / \mathrm{HeNJcl}$ inbrec. mouse strain (CLEA, Japan Inc., Tokyo). The eggs which were injected with HBV DNA were transferred to the ampulae of pseudopregnant females [Jcl:MCH (ICR) strain, CLEA, Japan Inc., Tokyo] and allowed to develop to term as pups (Fig. 2). The 3 transgenic mice were detected by Southern blot analysis of placenta and/or tail DNAs using nick-translated HBV as the probe. For Southern blot analysis of offsprings, total DNA was extracted as previously described."

Results and discussion. Of 12 animals ( $\mathrm{C} 3 \mathrm{H}$ ) born from the 2 pseudopregnant females, 3 were found positive for HBV sequences (Fig. 3). However, in spite of injection of the same BamHI fragment, the restriction patterns of integrated DNA differed among the 3 transgenic mice and were respectively 
designated as $\mathrm{H} 2-1 \mathrm{p}, \mathrm{H} 2-2 \mathrm{p}$ and $\mathrm{H} 2-5 \mathrm{~m}$. No hybridization to pure pBR327 DNA probe was observed. According to the restriction map analysis, a large deletion of the injected DNA had occurred in $\mathrm{H} 2-1 \mathrm{p}$ and $\mathrm{H} 2-5 \mathrm{~m}$ and the unit length (BamHI $5.4 \mathrm{~kb}$ fragment) was not retained (data not shown). On the other hand, in $\mathrm{H} 2-2 \mathrm{p}$, presence of the unit length following digestion with BamHI was presumed, since tandemly integrated copies of the integrated DNA were found. The $5.4 \mathrm{~kb}$ BamHI fragment that hybridized to HBV DNA most intensely contained multiple copies of the HBV sequences. The other fragments are likely to contain the junction between mouse and $\mathrm{HBV}$ genome and comprise the flanking DNA.
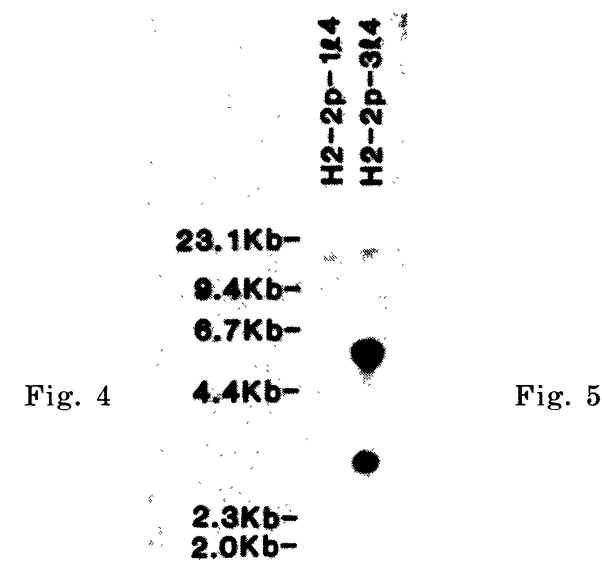

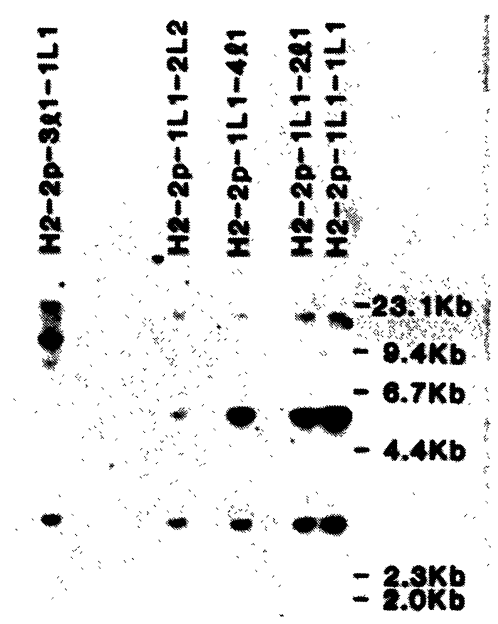

Figs. 4-5. 4: Southern blot analysis of tail DNAs of F1 descendants of the H2-2p lineage [(H2-2p-1l4( $q)$ and H2-2p-3l4( $\%)]$ after BamHI digestion. Positions of molecular weight markers of known length are shown on the left. 5: Southern blot analysis of tail DNAs of F2 descendants of the H2-p2 lineage [( H2-2p-3l11L1 ( o ) , H2-2p-1L1-2L2 ( o ), H2-2p-1L1-4l1 ( $)$ ), H2-2p-1L1-2l1 ( $~)$, and H2-2p1L1-1L1 ( $\delta$ )] after BamHI digestion. Positions of molecular weight markers of known length are shown on the right.

Descendants of the 3 new strains (H2-1p, H2-2p and $\mathrm{H} 2-5 \mathrm{~m}$ ) were produced by crossing to normal $\mathrm{C} 3 \mathrm{H}(+/+)$. The original integration pattern changed in only one out of $15 \mathrm{HBV}$ DNA positive F1 descendants of the H2-2p lineage, named H2-2p-1l4 as shown in Fig. 4. BamHI digestion revealed deletion of the unit length (BamHI $5.4 \mathrm{~kb}$ fragment) together with other bands and generation of new band of different size. No rearrangement was found in either F1 or $\mathrm{F} 2$ descendants of $\mathrm{H} 2-1 \mathrm{p}(0 / 20)$ and $\mathrm{H} 2-5 \mathrm{~m}(0 / 4)$. However, rearrangement was also found in one of the $5 \mathrm{HBV}$ DNA positive F2 descendants of H2-2p lineage (H2-2p-3l1-1L1) (Fig. 5). As with the F1 case, the rearrangement featured disappearance of the unit length (BamHI $5.4 \mathrm{~kb}$ fragment) and appearance of other additional bands of higher molecular weight. Furthermore, the intensity of the unit length BamHI $5.4 \mathrm{~kb}$ hybridization band differed among some animals of the H2-2p lineage (data not shown). These data strongly suggest that amplification of the unit length had occurred. However, we need more precise information to interpret this result, because there were already multiple copies in the original H2-2p. This does not detract, however, from the finding that 
transgenic mice of $\mathrm{H} 2-2 \mathrm{p}$ lineage are susceptible to DNA rearrangement. The fact that rearrangement was limited to the $\mathrm{H} 2-2 \mathrm{p}$ descendants indicates that the topology of H2-2p structure might be important for the DNA rearrangement. As mentioned above, the unit length BamHI fragment is capable of forming a stem and loop structure (Fig. 1), containing DR1 in the loop region. HBV contains two direct repeat sequences (DR1 and DR2) of 11 base pairs in the cohesive region. ${ }^{15)}$ Recent analysis of integrated HBV genome has shown that integration sometimes occurs in the DR1 region, indicating that these direct repeat sequences are preferred sites in the viral genome for recombination. ${ }^{10) .15}$ ) Moreover, the DR1 region is believed to be the origin of viral replication. ${ }^{16)}$

Rearrangement of injected DNA during integration in transgenic mouse eggs occurs fairly frequently and the phenomenon is not specific for the exogenous DNA employed. ${ }^{18)}$ The process may reflect an initially unstable DNA structure with subsequent attainment of a more stable unique conformation between donor DNA and cellular DNAs. In the reported rearrangements the donor DNA as well as the cellular sequences revealed duplications, deletions or changed organization. ${ }^{18)}$ Post-integration DNA rearrangements were found previously only in tumor tissues along with tumor formation in SV40, c-myc and bovine papillomavirus DNAs transgenic mice and have never been observed in normal tissues. ${ }^{19)-21)}$ The three pedigrees (H2-1p, H2-2p and $\mathrm{H} 2-5 \mathrm{~m}$ ) of transgenic mice were phenotypically normal during development and early adult life, at least within the first 6 months.

We are now investigating the mechanism of the observed rearrangements together with the effect of HBV DNA integration and subsequent rearrangement on the risk of HCC in the presence or absence of a chemical carcinogen. We anticipate that these transgenic mice may present a useful model system for studying the significance of viral integration in human hepatocarcinoma development, especially in terms of integration-associated aberrations of host DNA.

Acknowledgements. We thank Dr. Tatsuji Nomura for encouragement throughout this work. This work was supported by a grant-in-aid for Cancer Research and a grant-in-aid for special project research (Cancer-Bioscience) from the Ministry of Education, Science and Culture of Japan.

\section{References}

1) Beasley, R. P. (1982): Hepatology, 2, 21S-26S.

2) Shafritz, D. A. et al. (1981) : N. Engl. J. Med., 35, 1067-1073.

3) Hino, O. et al. (1984): Hepatology, 4, 90-95.

4) Hino, O. et al. (1984): Science, 225, 670-671.

5) Hino, O. et al. (1985): Int. J. Cancer, 35, 5-10.

6) Hino, O. et al. (1985): Proceedings of the Japanese Cancer Association, The 44th Annual Meeting, Tokyo, p. 46.

7) Fujisawa, J. et al. (1985): Proc. Natl. Acad. Sci. USA., 82, 2277-2281.

8) Koch, S. et al. (1984) : EMBO J., 3, 2185-2189.

9) Rogler, C. E. et al. (1985): Science, 230, 319-322

10) Hino, O. et al. (1986) : Proc. Natl. Acad. Sci. USA, 83, 8338-8342.

11) Cains, J. (1981): Nature (London), 289, 353-357.

12) Klein, G. (1981): ibid., 294, 313-318.

13) Dejean, A. et al. (1983) : Proc. Natl. Acad. Sci. USA., 82, 2505-2509.

14) Mizusawa, H. et al. (1985): ibid., 82, 208-212.

15) Tiollais, P. et al. (1985): Nature (London), 317, 489-495.

16) Seegar, C. et al. (1986) : Science, 232, 477-484.

17) Galloway, D. A. et al. (1984): Proc. Natl. Acad. Sci. USA., 81, 4736-4740.

18) Covarrubias, L. et al. (1986): ibid., 83, 6020-6024.

19) Brinster, R. L. et al. (1984): Cell, 37, 367-379.

20) Leder, A. et al. (1986) : ibid., 45, 485-495.

21) Lacey, M. et al. (1986): Nature (London), 322, 609-612. 